OPEN

SUBJECT AREAS:

ATMOSPHERIC

DYNAMICS

INNER PLANETS

Received

7 March 2014

Accepted

16 June 2014

Published

7 July 2014

Correspondence and requests for materials should be addressed to

X.J.Z. (xizheng@ xidian.edu.cn)

\section{Charge-to-mass Ratio of Saltating Particles in Wind-Blown Sand}

\author{
Tian-Li Bo ${ }^{1,2}$, Huan Zhang ${ }^{1,2}$ \& Xiao-Jing Zheng ${ }^{1,2,3}$
}

\begin{abstract}
${ }^{1}$ Key Laboratory of Mechanics on Western Disaster and Environment, Lanzhou University, Lanzhou, 730000, China, ${ }^{2}$ Department of Mechanics, Lanzhou University, Lanzhou, 730000, China, ${ }^{3}$ School of Electronical and Machanical Engineering, Xidian University, Xi'an, 710071, China
\end{abstract}

The electrification of sand particles plays an important role in aeolian events. In this paper, the charge-to-mass ratio vertical profiles of saltating particles in wind-blown sand were measured by a field experiments. By combining the results of field measurements with our previous wind-tunnel measurements, we discussed the factors affecting the charge-to-mass ratio of saltating particles. It reveals that the magnitude of the charge-to-mass ratio increases exponentially with height above the surface. In addition, the charge polarity of saltating particles depends on the relative size between saltating and creeping particles, and the magnitude of charge-to-mass ratio is determined by wind velocity and the relative size difference ratio between saltating and creeping particles.

$\mathrm{n}$ natural and industrial granular flows, the existence of electrostatic charges has been widely acknowledged ${ }^{1-6}$ and become an increasingly active area of research in recent years. The electrostatic charges can generate an intense electric field, and thus affect the transport and lifting of particles. For example, electric fields can affect the mass flux and saltation height of wind-blown sand ${ }^{2}$, the development of snow cornices in blowing snow ${ }^{4}$, and the sedimentation process of volcanic ash from eruption plumes ${ }^{5,6}$. On other planets, such as the Mars, the electric fields due to dust electrification in wind-blown sand, and other dusty phenomena can also affect the atmospheric chemistry and exploration ${ }^{7}$. Therefore it is necessary to make in-depth investigations on the electrification of granular systems.

In wind-blown sand, charge-to-mass ratio is usually used to characterize the sand electrification, which has importance for understanding the contact electrification of sand particles and determining the effects of electrostatic forces on the motion of saltating particles ${ }^{2,8}$. It can be obtained by measuring both the charge and mass of saltating particles collected in a Faraday cup during a certain period of time.

Although the electrification of wind-blown sand is well documented, the investigations of charge-to-mass ratio of saltating particles still remain poorly understand. On the one hand, previous wind-tunnel ${ }^{8-10}$ and field measurements ${ }^{1}$ found both positively and negatively charged saltating particles (as summarized in Table 1), and these studies did not explain why bipolar charged saltating particles exist. For example, Schmidt et al. ${ }^{1}$ first measured the average charge-to-mass ratio of saltating particles on the broad top of a dune, and found positively charged saltating particles with the average charge-to-mass ratio of $60 \mu \mathrm{c} / \mathrm{kg}$. However, later measurements in windtunnel by Zheng et al. ${ }^{8}$, Qu et al. ${ }^{9}$, and Zhang et al. ${ }^{10}$ respectively found that the average charge-to-mass ratios are in the range of $-124-0.95 \mu \mathrm{c} / \mathrm{kg},-304-158 \mu \mathrm{c} / \mathrm{kg}$, and $-24.3-65.8 \mu \mathrm{c} / \mathrm{kg}$. More recently, we measured the vertical profiles of charge-to-mass ratio in a wind-tunnel ${ }^{11}$ and found that the magnitude of average chargeto-mass ratio increase exponentially with height above the surface, with maximum value of $\sim 400 \mu \mathrm{c} / \mathrm{kg}$. On the other hand, previous studies also did not account for the effects of wind velocity and relative size difference between sand particles on the magnitude of charge-to-mass ratio. In fact, the magnitude of charge separation in the collisions between two particles depends on the relative particle size difference and the impacting velocity ${ }^{12,13}$. In wind-blown sand, the speed and diameter of saltating particles vary with height and wind velocity, and thus the charge-to-mass ratio must be affected by sand diameter and wind velocity.

In this study, the charge-to-mass ratio of saltating particles was measured by a field experiments. By combining the field measurements results and our previous wind-tunnel experimental data, we discussed the factors affecting the polarity of charge-to-mass ratio, and how the magnitude of charge-to-mass ratio depends on wind velocity and the relative difference in sand particle size. 
Table 1 | Summary experimental results of charge-to-mass ratios in wind-blown sand

\begin{tabular}{lccc} 
Study & Charge-to-mass ratio $(\mu \mathrm{c} / \mathrm{kg})$ & Diameter $(\mu \mathrm{m})$ & Wind Velocity $(\mathrm{m} / \mathrm{s})$ \\
\hline Schmidt et al. [1] (field) & 60 & 150 & $12 \mathrm{at} 1.5 \mathrm{~m}$ \\
Zheng et al. [8] (wind-tunnel) & $-124 \sim 0.95$ & $0 \sim 1000$ & $7 \sim 20$ at $0.45 \mathrm{~m}$ \\
Qu et al.[9] (wind-tunnel) & $-304 \sim 158$ & $80 \sim 315$ & $8 \sim 22 \mathrm{at} 0.3 \mathrm{~m}$ \\
Zhang et al. [10] (wind-tunnel) & $-24.3 \sim 65.8$ & $100 \sim 600$ & $8 \sim 22$ at $0.3 \mathrm{~m}$ \\
\hline
\end{tabular}

\section{Results and Discussion}

The field measurements were made on May 18, 2013 at the Surface Layer Turbulence and Environmental Science Test (SLTEST) facility in the Minqin, China. The configuration of the instrument array is depicted in Figure 1. Field measurements consisted of the wind velocity, charge-to-mass ratio, and the particle size distribution of saltating particles. The wind velocity was measured using sonic anemometers (Campbell Scientific CSAT3) manufactured by the USA Campbell company. The charge-to-mass ratios of saltating particles were measured by a simultaneous measurement system (CTMs), which is composed of a Faraday cup to receive sand particles, an electrometer (Keithley 6517B, whose measurement range and resolution are $2 \mu c$ and $10 p c$, respectively) to record real-time electric charges, a electronic balance to measure the mass of collected sand particles, and a computer to record data with the sampling frequency of $1 \mathrm{~Hz}$. That is, once saltating particles entrance into Faraday cup through input hole, electrometer and electronic balance can record the variation of the charge and mass of sand particles collected in the Faraday cup, and thus we can determine the variation of the charge-to-mass ratio of saltating particles. The particle size distribution of saltating sand particles was obtained by sand particle counter (SPC-91, Niigata Electric Co., Ltd.) with the sampling frequency of $1 \mathrm{~Hz}$. As the saltating particles pass through the slit between the transmitter and receiver laser beam, the SPC-91 detects the signal changes to measure the diameter from 30 to $500 \mu \mathrm{m}$ with 32 bin steps and the number of saltating particles of each bin. Four charge-to-mass ratio measurement systems were respectively mounted at $0.03,0.05,0.08$, and $0.10 \mathrm{~m}$ height above the surface, which spaced $0.3 \mathrm{~m}$ apart along a line perpendicular to the dominant wind direction. Four sand particle counter probes were installed in two SPC-91 sensors to obtain the particle size distribution at the measured heights of charge-to-mass ratio. Four sonic anemometers were mounted on anemometer tower to obtain wind velocity. In addition, the detailed description of our wind-tunnel measurements is given in Zhang et $\mathrm{al}^{11}$.

Figure 2 shows the vertical profiles of the charge-to-mass ratios of saltating particles for different wind velocities in the field and windtunnel measurements. First, form Figure 2 we can found that the magnitude of charge-to-mass ratios increases exponentially with height. Theoretical studies by Hu et al. ${ }^{12}$ and Bo et al. ${ }^{13}$ indicated that the charge-to-mass ratio increases with the impacting velocity of saltating particles, but decreases with the diameter of saltating particles. In Figure 3, we plot the particle size distributions of surface creeping particles and the saltating particles for different wind velocities. As shown in Figure 3, both in wind-tunnel and field measurements the mean diameters of saltating particles above $2 \mathrm{~cm}$ height are approximately constant with height. More recently, Farrell et al. ${ }^{14}$ analyzed the vertical distribution of saltating particles, and also found a inflection region between 0.05 and $0.15 \mathrm{~m}$ height above the surface, in which the mean diameter is invariant to changes in height. It means that the mean diameters may slightly affect the variation of charge-to-mass ratios with height within the saltation layer. In addition, the vertical profiles of saltating particle velocity for different wind velocities are shown in Figure 4 . As can be seen form Figure 3, with the height increasing the velocities of saltating particles increases exponentially. Therefore, it indicates that the variation of charge-to-mass ratios with height is mainly affected by the change in particle velocity.

Second, although the magnitude of charge-to-mass ratios increases exponentially with height in field and wind-tunnel experiments, its polarity is opposite. As shown by Figure 2, the charge-tomass ratio obtained by wind-tunnel experiments is positive, which is consistent with the measured results of Schmidt et al. ${ }^{1}$, and the charge-to-mass ratio obtained by field experiments is negative, which is also observed by other studies ${ }^{8-10}$. This discrepancy may be caused by two mechanisms: (1) the dependence of relative size between saltating particles and surface creeping particles on the particle size distribution of sand sample; (2) the size-dependent charge segregation of granular materials, i.e., larger and smaller particles tend to charge positively and negatively, respectively. For example, windtunnel measurements by Xing ${ }^{15}$ indicated that the size of saltating particles depend on the particle size distribution of sand sample. A numerical study by Dupont et al. ${ }^{16}$ also found that for mean sand diameter is $90 \mu \mathrm{m}$, the saltating particles are smaller than surface creeping particles; for mean sand diameter is $200 \mu \mathrm{m}$, the saltating particles are larger than surface creeping particles. This suggests that
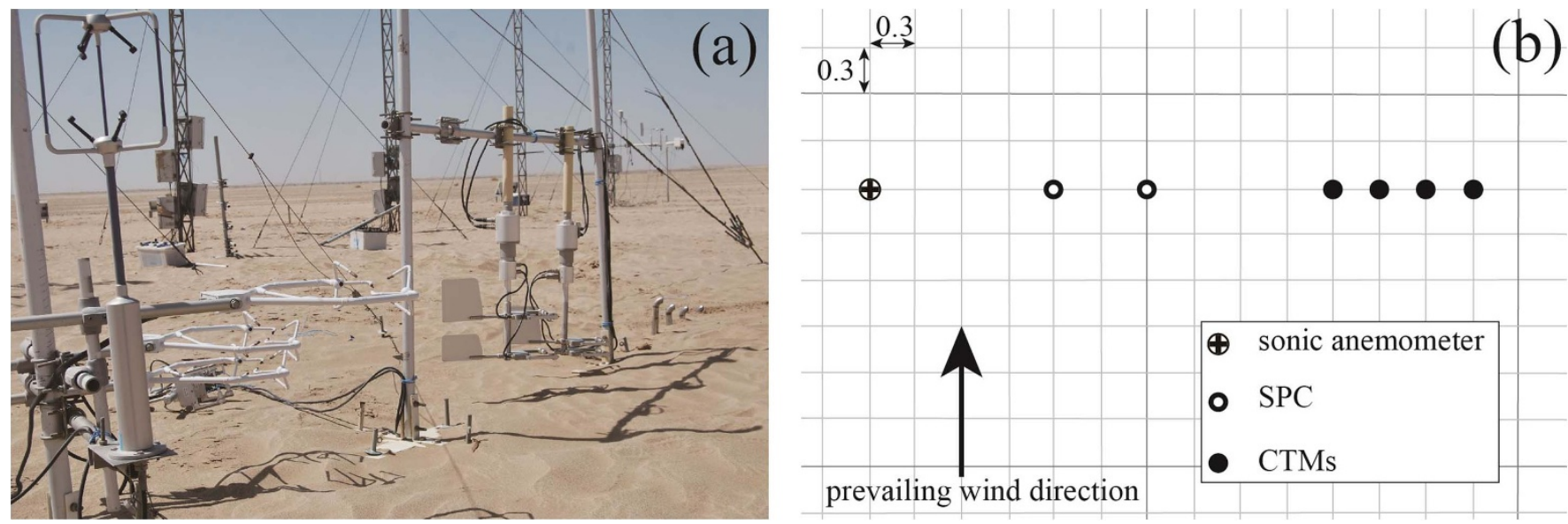

Figure $1 \mid$ (a) Oblique view of the instrument array at SLTEST. (b) Instrument array plan of deployment. Distance and coordinates in meters. 

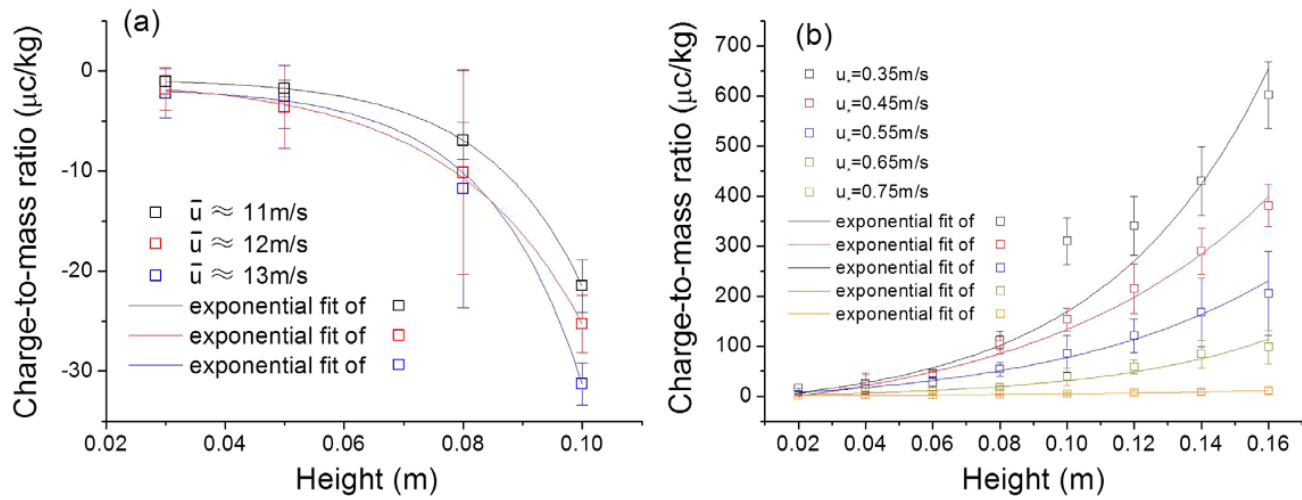

Figure $2 \mid$ Vertical profiles of charge-to-mass ratios for different wind velocities in (a) field and (b) wind-tunnel measurements. Here, dots correspond to experimental results, and lines correspond to exponential fit of measured results.
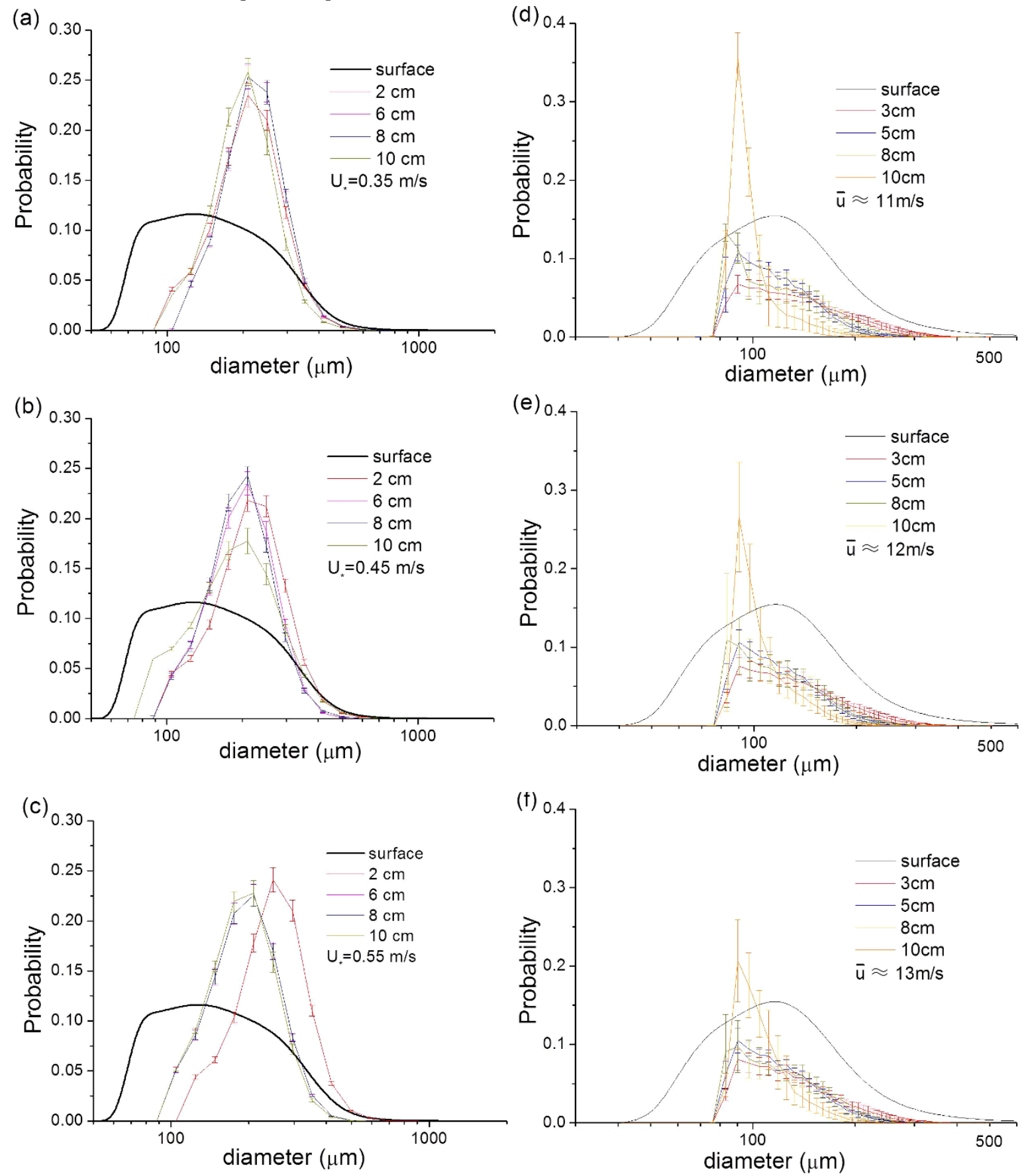

Figure 3 The particle size distribution of the saltating and creeping particles for different velocities in wind-tunnel (a-c) and field (d-f) experiments. 


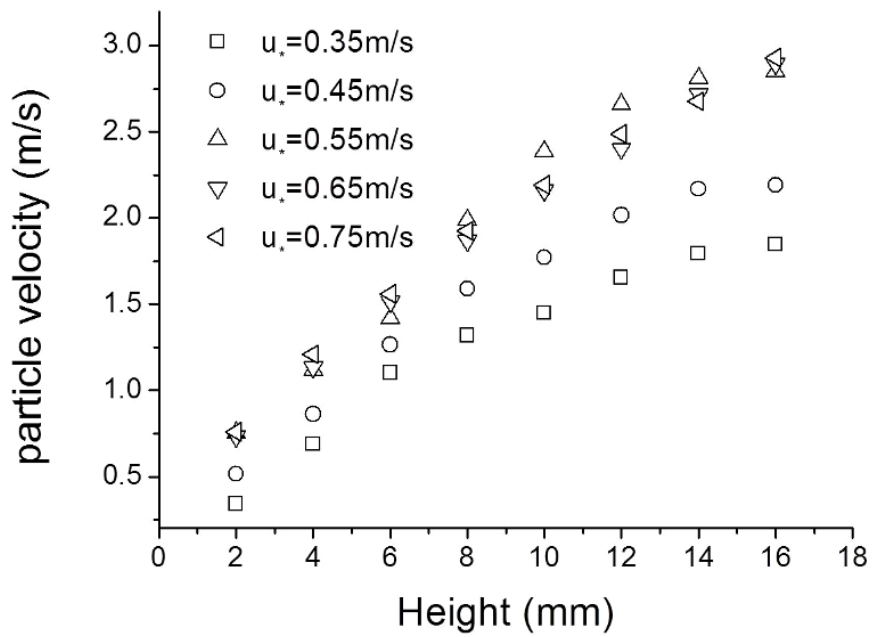

Figure 4 Vertical saltating particle velocity profiles for different wind velocities in wind-tunnel experiments.

for different particle size distributions of sand sample, the relative size between saltating and creeping particles is quite different. On the other hand, Forward et al. ${ }^{17}$ separately measured the particle size distributions of negatively and positively charged particles in a particle-particle interactions granular systems, and observed the sizedependent charge segregation phenomenon, namely, both negatively and positively charged particles are widely distributed, but the mean diameter of the negatively charged particles are smaller than the positively charged particles. In our wind-tunnel experiment, the mean diameter of saltating particles is $\sim 200 \mu \mathrm{m}$ larger than that of surface creeping particles $\sim 126 \mu \mathrm{m}$. However, in our field experiment, the mean diameter of saltating particles is $\sim 90 \mu \mathrm{m}$ smaller than that of surface creeping particles $\sim 117 \mu \mathrm{m}$. Therefore, according to the role of 'size-dependent charge segregation', the saltating particles are reasonability expected to charge positively and negatively in wind-tunnel and field experiments respectively, which is in agreement with our measured results.

Third, the magnitude of charge-to-mass ratios in wind-blown sand is larger than that in the field. As shown in Figure 2, the magnitude of charge-to-mass ratio is $\sim 30 \mu \mathrm{c} / \mathrm{kg}$ in the field, but is $\sim 600 \mu \mathrm{c} / \mathrm{kg}$ in wind-tunnel. A possible explanation for this is that compared with the field, there is a larger difference of mean diameters between saltating and creeping particles in wind-tunnel. The relative mean size difference ratio between saltating and creeping particles $d_{r}$ (defined as $d_{r}=\left|\left(d_{s}-d_{c}\right) / d_{c}\right| \times 100 \%$, where $d_{s}$ and $d_{c}$ are mean diameters of saltating and creeping particles, respectively) are respectively $58.7 \%$ and $23.1 \%$ in the wind-tunnel and field measurements. According to the theories proposed by Hu et al. ${ }^{12}$ and Bo et al. ${ }^{13}$, i.e., charge-to-mass ratios is proportional to the relative size difference ratio, and then the larger charge-to-mass ratio in wind-tunnel experiments can be interpreted by its larger relative size difference ratio compared to the field experiments.

Finally, the magnitude of charge-to-mass ratio decreases with wind velocity in wind-tunnel experiments but increases with wind velocity in field experiments. For the wind-tunnel experiment, the velocity of saltating particles increases with wind velocity (as shown in Figure 4), and above $2 \mathrm{~cm}$ height the variation of mean particle size with height is unobvious (as shown in Figure 5). This indicates that as wind velocity increases the decrease in charge-to-mass ratio may be mainly caused by the variation of particle velocity. In fact, the working section of wind-tunnel has limited length, and thus as the particle velocity increases the number of collisions between saltating and creeping particles decreases. Bo et al..$^{13}$ pointed out as the number of collisions increases the magnitude of charge-to-mass ratio first increase and then reach their equilibrium value after several thou-

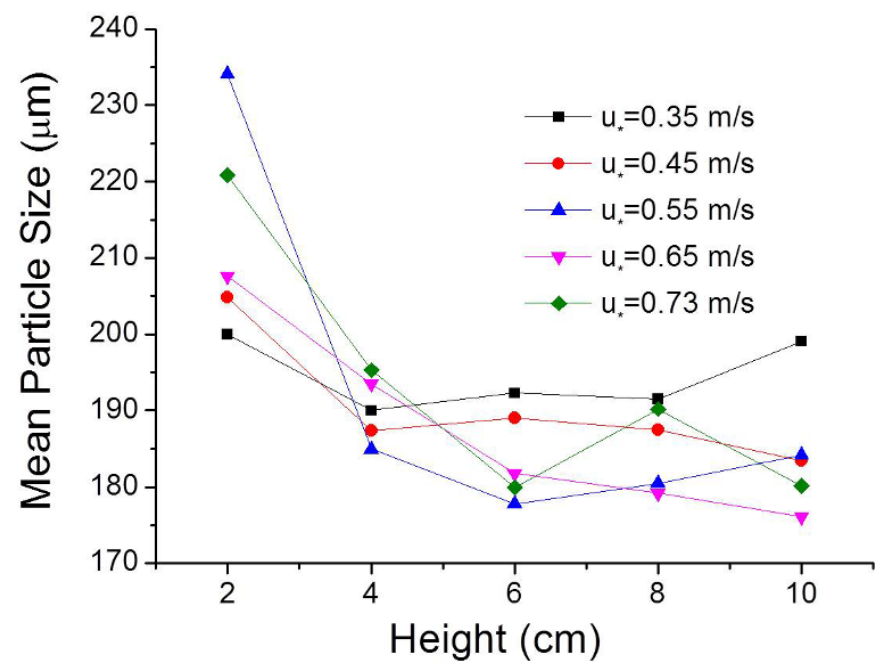

Figure 5 | Saltating particle diameter profiles for different wind velocities in wind-tunnel experiments.

sands times of collisions. Since the typical saltation length of saltating particles is $\sim 0.3 \mathrm{~m}^{18}$, the number of collisions is approximately 70 in our wind-tunnel experiments, which is much less than the number of collisions required for charge-to-mass ratio to reach equilibrium value. As wind velocity increases, sand particles velocity increases and thus the number of collisions decreases, which lead to the reduction in charge-to-mass ratio. For the field experiment since the transport of sand particles take place in an infinitely large spatial dimension, the influence of collision number on charge-to-mass ratio should be neglected. In addition, the variation of particle size with wind velocity is unobvious. Therefore, the increment in chargeto-mass ratio can be explained by the increasing particle velocity.

\section{Summary and Conclusion}

The electrification of sand particles is a universal phenomenon in wind-blown sand, and it plays an important role in the lifting and transport of sand particles. The charge-to-mass ratio of saltating particles is an important parameter to characterize the sand electrification. The previous measurements only obtained the average charge-to-mass ratio due to the limitation of experimental apparatus and conditions. Several numerical and theoretical studies ${ }^{2,19,20}$ attempt to explain the sand electrification, but little charge-to-mass ratio measured results are able to compare with their results.

In this paper, we measured the charge-to-mass ratio vertical profiles of saltating particles by a field experiments. By combining the field and our previous wind-tunnel experimental data, we discussed the factors affecting the polarity and magnitude of charge-to-mass ratio. Our results show that: 1) the magnitude of charge-to-mass ratio of saltating particles increases exponentially with the height above the surface; 2) the charge polarity of saltating particles depends on the relative size between saltating and creeping particles, for example, if the size of saltating particles is smaller than creeping particles, then the saltating particles charge negatively; 3 ) the magnitude of chargeto-mass ratio of saltating particles increase with the relative size difference ratio; 4 ) the magnitude of charge-to-mass ratio of saltating particles decreases with wind velocity in wind-tunnel but increases with velocity in field. Our measured results may be helpful to improve the understanding of sand electrification in wind-blown sand.

\section{Methods}

The field measurements were conducted on May 18, 2013 in the desert of Qingtu Lake, west of china (E:103 $40^{\prime} 03^{\prime \prime}, \mathrm{N}: 39^{\circ} 12^{\prime} 27^{\prime \prime}$ ). We measured the charge-to-mass ratio vertical profiles of saltating particles, wind velocity, and the particle size distribution of saltating particles simultaneously. The charge-to-mass ratios simultan- 
eous measurement system is developed by Lanzhou University, which determines the charge-to-mass ratio by measuring both the charge and mass of sand particles collected in a Faraday cup. The saltating particles arrive at the Faraday cup through a double-layer steel tube. The inner tube is insulated from the outer tube and connected to the Faraday cup by a coaxial cable, thus the influence of charge transfer between double-layer steel tube and sand particles can be neglected. A computer is used to record the charge and mass of sand particles collected in the Faraday cup. Therefore, the charge-to-mass ratio can be calculated as $\Delta q / \Delta m$ (where $\Delta q$ and $\Delta m$ are respectively the increment in charge and mass of collected sand particles). The particle size distribution of saltating sand particles was obtained by sand particle counter (SPC-91, Niigata Electric Co., Ltd.). SPC-91 records the diameter $d_{i}$ and the number of saltating particles $n_{i}$ of each particle bin, thus the probability of $i$-th particle bin is $n_{i} / \sum n_{i}$

1. Schmidt, D. S., Schmidt, R. A. \& Dent, J. D. Electrostatic force on saltating sand. J. Geophys. Res. 103, 8997-9001 (1998)

2. Kok, J. F. \& Renno, N. O. Electrostatics in Wind-Blown Sand. Phys. Rev. Lett. 100, 014501 (2008).

3. Schmidt, D. S., Schmidt, R. A. \& Dent, J. D. Electrostatic Force in Blowing Snow. Bound.-Layer Meteor. 93, 29-45 (1999).

4. Latham, J. \& Montagne, J. The possible importance of electrical force in the development of snow cornices. J Glaciol. 9, 375-384 (1970).

5. Miura, T., Koyaguchi, T. \& Tanaka, Y. Measurements of electric charge distribution in volcanic plumes at Sakurajima Volcano, Japan. Bull Volcanol. 64, 75-93 (2002).

6. Mather, T. A. \& Harrison, R. G. Electrification of volcanic plumes. Surv Geophys. 27, 387-432 (2006).

7. Renno, N. O. \& Kok, J. F. Electrical activity and dust lifting on Earth, Mars, and beyond. Space Sci. Rev. 137, 419-434 (2008).

8. Zheng, X. J., Huang, N. \& Zhou, Y. H. Laboratory measurement of electrification of wind-blown sands and simulation of its effect on sand saltation movement. J. Geophys. Res. 108, 4322 (2003).

9. Qu, J. J., Yan, M. H., Dong, G. R., Zhang, H. F., ZU, R. P., Tuo, W. Q., Zhao, A. G., Xiao, Z. H., Li, F. \& Yang, B. Wind tunnel simulation experiment and investigation on the electrification of sandstorms. Sci. China, Ser. D 47, 529-539 (2004).

10. Zhang, H. F., Wang, T., Qu, J. J. \& Yan, M. H. An experimental and observational study on the electric effect of sandstorms. Chin. J. Geophys. 47, 53-60 (2004).

11. Zhang, H., Zheng, X. J. \& Bo, T. Electrification of saltating particles in wind-blown sand: Experiment and theory. J. Geophys. Res. Atmos. 118 (2013).

12. Hu, W., Xie, L. \& Zheng, X. J. Contact charging of silica glass particles in a single collision. Appl. Phys. Lett. 101, 114107 (2012).

13. Bo, T. L., Zhang, H., Hu, W. W. \& Zheng, X. J. The analysis of electrification in windblown sand. Aeolian Research 11, 15-21 (2013).
14. Farrell, E. J., Sherman, D. J., Ellis, J. T. \& Li, B. Vertical distribution of grain size for wind blown sand. Aeolian Research 11, 51-61 (2012).

15. Xing, $M$. The harmonious character in equilibrium aeolian transport on mixed sand bed. Geomorphology 86, 230-242 (2007).

16. Dupont, S., Bergametti, G., Marticorena, B. \& Simoens, S. Modeling saltation intermittency. J. Geophys. Res. 118 (2013)

17. Forward, K. M., Lacks, D. J. \& Sankaran, R. M. Particle-size dependent bipolar charging of Martian regolith stimulant. Geophys. Res. Lett. 36, L13201 (2009).

18. Kok, J. F., Parteli, E. J. R., Michaels, T. I. \& Karam, D. B. The physics of wind-blown sand and dust. Rep. Prog. Phys. 75, 106901 (2012).

19. Kok, J. F. \& Lacks, D. J. Electrification of granular systems of identical insulators. Phys. Rev. E 79, 051304 (2009).

20. Hu, W. W., Xie, L. \& Zheng, X. J. Simulation of the electrification of wind-blown sand. Eur. Phys. J. E 35, 22 (2012).

\section{Acknowledgments}

This research was supported by a grant from the National Natural Science Foundation of China (Nos. 11072097, 11232006, 11202088, 10972164 and 11121202), the Science Foundation of Ministry of Education of China (No. 308022), Science and technology project of Gansu Province (No. 1208RJYA006) and National Key Technology R\&D Program (No. 2013BAC07B01). The authors express their sincere appreciation to the support.

\section{Author contributions}

T.B. and X.Z. wrote the main manuscript text and H.Z. prepared figures 1-5. T.B. and X.Z. designed and supervised the experiments. The experiments were performed and analyzed by H.Z. All authors reviewed the manuscript

\section{Additional information}

Competing financial interests: The authors declare no competing financial interests.

How to cite this article: Bo, T.-L., Zhang, H. \& Zheng, X.-J. Charge-to-mass Ratio of Saltating Particles in Wind-Blown Sand. Sci. Rep. 4, 5590; DOI:10.1038/srep05590 (2014).

This work is licensed under a Creative Commons Attribution-NonCommercialNoDerivs 4.0 International License. The images or other third party material in this article are included in the article's Creative Commons license, unless indicated otherwise in the credit line; if the material is not included under the Creative Commons license, users will need to obtain permission from the license holder in order to reproduce the material. To view a copy of this license, visit http:// creativecommons.org/licenses/by-nc-nd/4.0/ 